\title{
Tauopathy-Associated Tau Fragment Ending at Amino Acid 224 Is Generated by Calpain-2 Cleavage
}

Cicognola $\mathrm{C}^{1}$, Satir $\mathrm{TM}^{2}$, Brinkmalm $\mathrm{G}^{1}$, Matečko-Burmann $\mathrm{I}^{3}$, Agholme $\mathrm{L}^{2}$, Bergström $\mathrm{P}^{2}$, Becker $\mathrm{B}^{1,4}$, Zetterberg $\mathrm{H}^{1,2,4,5,6}$, Blennow $\mathrm{K}^{1,4}$, Höglund $\mathrm{K}^{1,4}$

1. Department of Psychiatry and Neurochemistry, Institute of Neuroscience and Physiology, The Sahlgrenska Academy at University of Gothenburg, Mölndal, Sweden.

2. Department of Psychiatry and Neurochemistry, Institute of Neuroscience and Physiology, The Sahlgrenska Academy at the University of Gothenburg, Gothenburg, Sweden.

3. Department of Psychiatry and Neurochemistry, Wallenberg Centre for Molecular and Translational Medicine, University of Gothenburg, Gothenburg, Sweden.

4. Clinical Neurochemistry Laboratory, Sahlgrenska University Hospital, Mölndal, Sweden.

5. Department of Neurodegenerative Disease, UCL Institute of Neurology Queen Square, London, UK.

6. UK Dementia Research Institute at UCL, London, UK. 


\begin{abstract}
BACKGROUND:

Tau aggregation in neurons and glial cells characterizes tauopathies as Alzheimer's disease (AD), progressive supranuclear palsy (PSP), and corticobasal degeneration (CBD). Tau proteolysis has been proposed as a trigger for tau aggregation and tau fragments have been observed in brain and cerebrospinal fluid (CSF). Our group identified a major tau cleavage at amino acid (aa) 224 in CSF; N-terminal tau fragments ending at aa 224 (N-224) were significantly increased in $\mathrm{AD}$ and lacked correlation to total tau (t-tau) and phosphorylated tau (p-tau) in PSP and CBD.
\end{abstract}

\title{
OBJECTIVE:
}

Previous studies have shown cleavage from calpain proteases at sites adjacent to aa 224. Our aim was to investigate if calpain- 1 or -2 could be responsible for cleavage at aa 224 .

\section{METHODS:}

Proteolytic activity of calpain-1, calpain-2, and brain protein extract was assessed on a custom tau peptide (aa 220-228), engineered with fluorescence resonance energy transfer (FRET) technology. Findings were confirmed with in-gel trypsination and mass spectrometry (MS) analysis of brain-derived bands with proteolytic activity on the FRET substrate. Finally, knockdown of the calpain-2 catalytic subunit gene (CAPN2) was performed in a neuroblastoma cell line (SH-SY5Y).

\section{RESULTS:}

Calpain-2 and brain protein extract, but not calpain-1, showed proteolytic activity on the FRET substrate. MS analysis of active gel bands revealed presence of calpain-2 subunits, but not calpain-1. Calpain-2 depletion and chemical inhibition suppressed proteolysis of the FRET substrate. CAPN2 knock-down caused a $76.4 \%$ reduction of $\mathrm{N}-224$ tau in the cell-conditioned media.

\section{CONCLUSIONS:}

Further investigation of the calpain-2 pathway in the pathogenesis of tauopathies is encouraged. 


\section{Background}

Tauopathies are a group of diseases characterized by the build-up of tau protein aggregates in neurons and glial cells including, among others, Altheimer's disease (AD) and disorders characterized by frontotemporal lobar degeneration (FTLD), such as progressive supranuclear palsy (PSP) and corticobasal degeneration (CBD). Tau is a neuronal protein involved in microtubule stability, whose function is regulated by a rigorous phosphorylation/dephosphorylation mechanism, with the tubulin-binding capacity of tau being activated in the dephosphorylated state and inhibited in the phosphorylated state [1]. For many years, tau hyperphosphorylation was considered the main mechanism behind the formation of tau aggregates, through a multi-step process [2]. More recently, tau proteolysis is gaining increasing attention due to its observed ability to trigger tau aggregation in neuronal cell models [3]. Several tau fragments derived from cleavage at different sites have been observed in tauopathy brains, but not all the proteases responsible for their production have been identified (reviewed in [4]).

Tau fragmentation is also reflected in the cerebrospinal fluid (CSF), with several studies suggesting that tau is present as different fragments in CSF, with $\mathrm{N}$-terminal and mid-region tau representing the most abundant variants and showing significantly higher concentrations in $\mathrm{AD}$ compared to controls when measured with immunoassays [5-15]. Similar results were also observed in plasma with ultrasensitive Single molecule array (Simoa)-based assays, showing significant differences in $\mathrm{N}$-terminal tau concentration in CSF between patients with AD dementia and mild cognitive impairment (MCI) due to AD versus controls [15].

It should be noted that the aforementioned assays do not measure tau fragments of a specific length; the antibodies used are not neo-epitope-specific but bind to epitope regions of several amino acids and, if the epitope is present, the assays are not able to differentiate between longer or shorter tau fragments or full-length tau. Our group has recently published a study where immunoprecipitation mass spectrometry (IP-MS) was used to identify naturally occurring endogenous tau fragments in CSF [16]. A major pool of tau consisted of fragments truncated at aa 224, and tau fragments going from the N-terminal to aa $224(\mathrm{~N}-224)$ were shown to be significantly increased in AD cohorts compared to controls. Tau truncated at aa 224 was also observed at tangle level in immunohistochemistry experiments. The results suggested a diseaserelated upregulated cleavage at this site, which leads to enhanced release from neurons of the $\mathrm{N}$-terminal fragment and, ultimately, higher CSF concentration of the fragment and a deposition into tangles. Also, concentrations of the fragment were extremely low in PSP and CBS cohorts, as the ones of t-tau and p-tau, but N-224 lacked correlation to p-tau and t-tau, suggesting a different underlying disease mechanism compared to $\mathrm{AD}$, which does not include cleavage of $\mathrm{N}$-terminal tau or its secretion into the CSF from brain (ref again).

While some of the proteases responsible for tau cleavage at the $\mathrm{N}$-terminus have been identified, the one responsible for cleavage at aa 224 is currently unknown. An N-terminal tau fragment of $17 \mathrm{kDa}$, spanning aa 45-230, was observed in AD, PSP and CBD brain [17, 18]. The fragme nt was cleaved in vitro by proteases of the calpain family, although it is not clear if calpain- 1 or 2 was responsible for the cleavage at aa $230[18,19]$. Also, a $24 \mathrm{kDa} \mathrm{C}$-terminal fragment of tau, produced by calpain- 1 cleavage at aa 242 , was observed in AD and FTLD brain with tau mutation and showed seeding and propagation activity in vitro [20].

Calpains belong to a family of cysteine proteases activated by calcium and inhibited by calpastatin [21-23]. The two most common isoforms are calpain-1 and -2 , which have differe nt calcium concentration requirements for activation: calpain-1 (also called $\mu$-calpain) is activated 
by micromolar concentrations of calcium, while calpain-2 (m-calpain) needs millimolar concentrations [24]. Calpain-1 and -2 are heterodimers with a similar structure and share a common regulatory subunit of $28 \mathrm{kDa}$ encoded by the CAPNS1 gene, but have different catalytic subunits, both $80 \mathrm{kDa}$, encoded by $C A P N 1$ and $C A P N 2$, respectively [24]. Calpain- 1 and -2 are constitutionally expressed and are implicated in synaptic plasticity with different and sometimes opposite roles [25]. Most importantly, calpains have consistently been shown to be involved in neurodegeneration mechanisms, such as hyperphosphorylation and cleavage of tau, and increased concentrations of calpain- 1 have been found in AD brains [26-31]. These observations made the calpain system the object of increasing attention as a possible therapeutic target for different neurodegenerative diseases [32].

Since tau ending at aa 224 showed potential as a tauopathy biomarker, our aim was to identify the enzyme responsible for cleavage at this site, as it could have potential implications in elucidating disease mechanisms and possibly developing therapeutics. Since previous literature had shown proteolytic activity of calpain proteases in the region surrounding the aa 224 cleavage site, we tested commercially available recombinant calpain-1 and in-house-produced calpain-2 as candidate enzymes for the cleavage, using a recombinant custom peptide containing the cleavage site at 224 and fluorescence resonance energy transfer (FRET) technology. We confirmed our findings in brain protein extracts using MS analysis and in a SHSY5Y knock-down model.

\section{Materials and methods}

\section{Preparation of brain extract}

Brain tissue was kindly supplied by the Netherlands Brain Bank. Brains were pathologically diagnosed as $\mathrm{AD}$ and controls and assessed with Braak staging (Tab. 1) [33]. Samples were collected 4-7 hours post-mortem from different regions of the parietal lobe and stored at $-80^{\circ} \mathrm{C}$. Upon homogenization, brain material was kept on dry ice and two different protocols were applied. In protocol 1, $100 \mathrm{mg}$ samples were excised and put in TBS solution (20 mM Tris, 137 $\mathrm{mM} \mathrm{NaCl}, \mathrm{pH}$ 7.6) with phosphatase inhibitor (PhosphoStop®, Sigma Aldrich) and homogenized with a motor homogenizer to final a tissue:TBS ratio of 1:5. In protocol 2, the original sample weight was $200 \mathrm{mg}$ and the initial dilution was 1:2.5. Samples from both protocols were further diluted in TBS solution up to $2 \mathrm{~mL}$, transferred to polypropyle ne centrifuge tubes (Bell-top Quick Seal centrifuge tubes, Beckman Coulter) and then ultracentrifuged for 20 minutes at $200000 \mathrm{x} \mathrm{g}, 4^{\circ} \mathrm{C}$. Supernatants were collected, aliquoted and stored at $-80^{\circ} \mathrm{C}$ pending analysis. In protocol 1 , the pellet from the centrifugation in TBS was also resuspended in a solution of $1 \%$ sarkosyl and ultracentrifuged for 20 minutes at $200000 \mathrm{x}$ $\mathrm{g}$, at room temperature. The resulting pellet was also re-homogenized and centrifuged in the same way. The supernatants from the two different steps were pooled, aliquoted and stored at $80^{\circ} \mathrm{C}$ pending analysis. 


\begin{tabular}{|l|l|l|l|l|l|l|}
\hline Sample & Region & $\begin{array}{l}\text { Braak } \\
\text { stage }\end{array}$ & Sex & Age & $\begin{array}{l}\text { Time post-mortem } \\
\text { (hh:mm) }\end{array}$ & Protocol \\
\hline AD 1 & $\begin{array}{l}\text { Superior } \\
\text { parietal gyrus }\end{array}$ & 5 & $\mathrm{~F}$ & 73 & $04: 25$ & 1 \\
\hline CTRL 1 & $\begin{array}{l}\text { Inferior } \\
\text { parietal gyrus }\end{array}$ & 0 & $\mathrm{~F}$ & 63 & $06: 25$ & 1 \\
\hline AD 2 & $\begin{array}{l}\text { Superior } \\
\text { parietal gyrus }\end{array}$ & 6 & $\mathrm{M}$ & 72 & $05: 30$ & 2 \\
\hline AD 3 & $\begin{array}{l}\text { Superior } \\
\text { parietal gyrus }\end{array}$ & 6 & $\mathrm{~F}$ & 76 & $05: 30$ & 2 \\
\hline CTRL 2 & $\begin{array}{l}\text { Superior } \\
\text { parietal gyrus }\end{array}$ & 0 & $\mathrm{~F}$ & 61 & $06: 50$ & 2 \\
\hline CTRL 3 & Parietal cortex & 1 & $\mathrm{M}$ & 61 & $05: 40$ & \\
\hline
\end{tabular}

Table 1: description of brain samples included in the study.

\section{Proteases, inhibitor, antibodies and FRET substrate}

Calpain-1 was derived from human erythrocytes (\#208713, Millipore, $1.68 \mathrm{mg} / \mathrm{mL}$, specific activity 2069 units/mg). For inhibition, we used E-64c calpain inhibitor (\#E0514, Sigma Aldrich). Anti-calpain-2 antibody was a mouse monoclonal IgG1 (kappa light chain) specific for an epitope between amino acids 2-27 at the N-terminus of human calpain-2 (E-10, sc373966, Santa Cruz Biotechnology). Secondary antibody for western blot was a mouse IgG kappa-binding protein (m-IgGK BP) conjugated to HRP (sc-516102, Santa Cruz Biotechnology). The custom FRET substrate with the tau sequence including aa 224 was bound on the $\mathrm{N}$-terminus to a fluorophore and on the $\mathrm{C}$-terminal to a quencher molecule (sequence: 5FAM-TREPKKVAVV(QXL520), Anaspec, Eurogentec). The lyophilized substrate was reconstituted in $2 \mathrm{~mL}$ of ultrapure water, $1 \%$ DMSO and ammonium hydroxide $1 \%(0.7 \mathrm{mM})$.

\section{Calpain-2 and empty vector expression and cell culture}

The pET_Duet-1 vector containing the different calpain constructs, CAPN2 and CAPNS1_CAPN2, as well as puc19 as a negative control, was transformed into BL21_RPCodonPlus competent cells (Agilent), plated on LB-Amp plate $(50 \mu \mathrm{g}$ ampicillin/mL) and incubated over night at $37^{\circ} \mathrm{C}$. For expression, several colonies from each construct were picked for inoculation of overnight culture in LB. The overnight culture was diluted 1:100 in $50 \mathrm{~mL}$ $\mathrm{LB}$ and grown at $37^{\circ} \mathrm{C}$. When $\mathrm{OD} 600$ reached $0.5-0.8$, protein expression was induced with 0.5 $\mathrm{mM}$ IPTG and cultures were grown for 5 hours or overnight at $25^{\circ} \mathrm{C}$. Five $\mathrm{mL}$ from each culture was saved as crude supernatant at $-80^{\circ} \mathrm{C}$, the remaining culture was centrifuged at $6000 \mathrm{rpm}$ for 15 minutes at $4^{\circ} \mathrm{C}$ and the cleared supernatant and pellet were stored at $-80^{\circ} \mathrm{C} .1-2 \mathrm{~mL}$ of lysis buffer (20 mM Tris, $150 \mathrm{mM} \mathrm{NaCl}, 1 \% \mathrm{NP} 40, \mathrm{pH}$ 7.4) were added to each pellet from a 100 $\mathrm{mL}$ culture and incubated for 30 minutes. Samples were then centrifuged at 12000 RPM for 20 minutes and the supernatant was collected for analysis.

\section{Alternate calpain-2 expression and purification}


E. coli BL21( $\lambda$ DE3) Star (Novagen) were transformed with a pETDuet1 plasmid harboring truncated $\triangle \mathrm{N} 25 \mathrm{CAPNS} 1$ and CAPN2 containing a C-terminal His Tag. Transformed cells were spread on LB-Agar plates containing ampicillin $(100 \mu \mathrm{g} / \mathrm{mL})$ (Sigma Aldrich) and grown overnight at $37^{\circ} \mathrm{C}$. For protein expression and purification, the cells were pre-cultured overnight in M9 medium 1 (containing $100 \mu \mathrm{g} / \mathrm{mL}$ ampicillin, $30^{\circ} \mathrm{C}$ ). The main culture was inoculated with pre-cultured medium to OD $600=0.1$ and grown at $30^{\circ} \mathrm{C}$ until a cell density of OD $600 \sim$ 0.8-1.0 was reached. Protein expression was induced by addition of $0.2 \mathrm{mM}$ isopropylthiogalactoside (IPTG) (Thermo Scientific) at $20^{\circ} \mathrm{C}$ for $16 \mathrm{~h}$. Cells were harvested by centrifugation at $5000 \mathrm{xg}$ at $4^{\circ} \mathrm{C}$ for 20 minutes. The resulting cell pellet was washed once with ice-cold TED buffer (20mM Tris-Cl pH 7.5, 1mM EDTA, $1 \mathrm{mM}$ DTT), resuspended in $50 \mathrm{~mL}$ ice-cold TED buffer and lysed by four passes through an Emulsiflex (Avestin).

The lysate was centrifuged at $22000 \mathrm{x}$ g for 40 minutes. The supernatant was applied to a HiTrap Q HP (GE Healthcare) column equilibrated with TED buffer, washed with 5 column volume s TED buffer and eluted by a linear gradient from $0-400 \mathrm{mM} \mathrm{NaCl}$ in TED buffer. The fractions containing the calpain-2 complex were pooled and $\mathrm{NaCl}$ and $\mathrm{MgCl}_{2}$ were added to final concentrations of $0.5 \mathrm{M}$ and $5 \mathrm{mM}$, respectively. The pooled fractions were loaded on a HisTrap HP column (GE Heathcare), equilibrated with buffer A (20mM NaPi buffer 7.8, $0.5 \mathrm{M} \mathrm{NaCl}$, $1 \mathrm{mM}$ DTT), washed with 5 column volumes of the same buffer containing $50 \mathrm{mM}$ imidazol and eluted with a linear gradient from $50 \mathrm{mM}-0.3 \mathrm{M}$ imidazol. To the eluting fractions, immediately $1 \mathrm{mM}$ EDTA was added. Proteolytically active fractions were immediately dialysed against TED buffer. The purity of the proteins was analyzed by SDS page. The proteolytic activity of calpain-2 was measured using casein (Sigma Aldrich), as described before $[34,35]$.

\section{Generation of in house anti-tau 224 antibody}

Anti-tau 224 was generated by immunization of 8 weeks old Balb/c mice with KLH-conjugated recombinant tau 224 peptide (KLH)-CGGGRTPSLPTPPTREPK (Caslo) in complete Freund's adjuvant (Sigma Aldrich). After two-three dosages with the recombinant protein fragment (approximately $75 \mu \mathrm{g} /$ mouse), the spleen was removed and B-cells were fused with the myeloma cell line SP2/0 following standard procedures. Approximately 10 days after fusion, cell media were screened for the antibodies using full-length recombinant tau and the recombinant protein fragment. Clones that reacted with the recombinant protein fragments but not with full length tau and negative control protein were further grown, subcloned and subsequently frozen in liquid nitrogen. The isotype was determined using a commercially available kit (Pierce Rapid Isotyping Kit-Mouse). Finally, the antibody was purified using a protein G column (GE Healthcare).

\section{SHSY5Y cell culture}

SHSY5Y cells (ECACC; Sigma Aldrich, St. Louis, MO, USA) were cultured in DMEM-F12 Glutamax medium supplemented with $10 \%$ fetal bovine serum (Corning ${ }^{\mathrm{TM}}$, FBS), $1 \%$ Penicillin-Streptomycin (Gibco ${ }^{\mathrm{TM}}$ ) (plating media), at $37^{\circ} \mathrm{C}$, humidified air with $5 \% \mathrm{CO}_{2}$. The medium was changed twice a week and the cells were passaged at $80 \%$ confluency. In order to initiate the neuronal differentiation, cells were harvested with $0.05 \%$ Trypsin-EDTA $\left(\right.$ Gibco $^{\mathrm{TM}}$, phenol red) and plated to a final density of 500000 cells/ T75 culture flask in the plating medium supplemented with all-trans retinoic acid (RA, $10 \mu \mathrm{M}$ final concentration) for 6-7 days. 


\section{Transfection}

The last day of the differentiation (day 6 or 7), cells were trypsinated and plated either onto 24well plates or 6-well plates (Corning ${ }^{\circledR}$ ) in the plating media supplemented with RA to a final density of 24000 cells $/ \mathrm{cm}^{2}$. The following day, 30 minutes prior to the transfection, the plating medium was replaced with fresh antibiotics-free DMEM-F12-Glutamax supplemented with RA (transfection media). Lipofectamine ${ }^{\circledR}$ RNAiMAX reagent (Invitrogen) and siRNA targeting human calpain-2 (Silencer®, siRNA ID: s320, cat\#4390825) and scrambled control (Stealth RNAi $^{\mathrm{TM}}$ siRNA Negative Control High GC Duplex) were prepared according to the manufacturer's protocol, with minor changes. Briefly, $1.5 \mu \mathrm{L} / \mathrm{cm}^{2}$ Lipofectamine and 1 $\mathrm{pmol} / \mathrm{cm}^{2}$ silencer/scrambled control were diluted in DMEM $(150 \mu \mathrm{L}$ for 6-well plate and 50 $\mu \mathrm{L}$ for 24-well plate) in separate sterile tubes. Then tubes containing Lipofectamine and nucleic acids were mixed 1:1 and incubated 5 minutes at RT. After the incubation, transfection complex added into the wells containing the RA-differentiated cells. After 48 hours from the first transfection, the media were replaced with fresh transfection medium and the second transfection, performed using the same conditions, was performed. 48 hours after the second transfection, the media were replaced with fresh transfection medium and the cells were kept 48 hours in the incubator. Cell pellet and cell-conditioned media were collected and kept in -80 ${ }^{\circ} \mathrm{C}$ for further analysis.

\section{Cell lysis protocol}

The cell pellets collected from transfected cells were lysed following a protocol previously described [36]. Briefly, cells were lysed in RIPA buffer (20 mM Tris-HCl, pH 7.5, $150 \mathrm{mM}$ $\mathrm{NaCl}, 1 \mathrm{mM}$ EDTA, $1 \%$ Triton X-100, 0.5\% deoxycholate $0.1 \%$ SDS) supplemented with MiniComplete protease inhibitor cocktail (Roche), and sonicated for 10 minutes at $4^{\circ} \mathrm{C}$ and incubated on ice for 20 minutes. Samples were then centrifuged at $4000 \mathrm{x} \mathrm{g}, 5$ minutes at $4^{\circ} \mathrm{C}$. The supernatants were then transferred to fresh tubes and stored at $-80^{\circ} \mathrm{C}$ until further analysis.

\section{Total RNA extraction and cDNA synthesis}

The pellet from the transfected cells were lysed in $200 \mu \mathrm{L}$ RLT Buffer supplemented with 4 $\mathrm{mM}$ dithiothreitol (Sigma-Aldrich) [34]. Total RNA was extracted and purified on a QiaCube robotic work station (Qiagen), using the RNeasy Mini protocol according to manufacturer's instructions. Total RNA concentration was measured on a Nano Drop 2000/2000c spectrophotometer (Thermo Scientific) and diluted in RNase-free water. cDNA was synthesized using a High Capacity cDNA kit with RNase inhibitor (Applied Biosystems) in a total reaction volume of $20 \mu \mathrm{L}$ and converted in a single-cycle reaction on a 2720 Thermal Cycler (Applied Biosystems): $25^{\circ} \mathrm{C}$ for 10 minutes, $37^{\circ} \mathrm{C}$ for 120 minutes and $85^{\circ} \mathrm{C}$ for 5 minutes.

\section{Quantitative PCR (qPCR)}

Quantitative PCR (qPCR) was performed using inventoried TaqMan Gene Expression Assays with FAM reporter dye in TaqMan Universal PCR Master Mix with UNG according to manufacturer's protocol, in a total reaction volume of $25 \mu \mathrm{L}$. qPCRs were carried out on MicroAmp 96-well optical microtiter plates on a 7900HT Fast QPCR System (Applied Biosystems), using standard settings for Standard Curve qPCR. TaqMan Gene Expression Assays for the following genes were used: calpain-1 (Hs00559804_ml), calpain-2 (Hs00965097_m1), MAPT 
(microtubule associated protein tau) (Hs00902194_m1) and as the reference gene POLR2a (Hs00172187_m1). All samples were run in duplicates. PCR results were then analyzed with the SDS 2.3 software (Applied Biosystems) and the relative quantity of gene expression was determined using the $\triangle \triangle \mathrm{CT}$ method [36], with scrambled transfected cells as the calibrator and CT:s of POLR2a as the endogenous reference.

\section{Detection of the fluorescence from cleavage of quenched FRET substrate}

Calpain-1 was tested at several dilutions similarly to a protocol previously described [37]. Calpain- 1 was diluted in $50 \mathrm{mM}$ Tris- $\mathrm{HCl} \mathrm{pH} 7.5,300 \mu \mathrm{M} \mathrm{CaCl} 2(1 / 40,1 / 120,1 / 360,1 / 1080)$. Positive control and negative control were respectively FRET substrate combined with $1 \mu \mathrm{L}$ trypsin solution $(0.1 \mu \mathrm{g} / \mu \mathrm{L}$, V511A, Promega) or with $40 \mu \mathrm{L}$ assay buffer (blank). Cell lysates from calpain-2 and empty vector cultures (negative control) were spiked with $\mathrm{CaCl}_{2}$ to a final concentration of $5 \mathrm{mM}$ and run neat.

The FRET substrate was brought to a final concentration of $7 \mu \mathrm{M}$ in assay buffer. Forty $\mu \mathrm{L}$ of enzyme and $40 \mu \mathrm{L}$ of substrate were mixed in a 384-well plate (AlphaPlate-384, PerkinElmer). The development of fluorescence was observed on a Spectramax Gemini reader (wavelength $485 \mathrm{~nm}$, emission $520 \mathrm{~nm}$, cut-off $495 \mathrm{~nm}$, Molecular Devices) over 45 minutes, with measurements recorded every two minutes.

\section{Immunoprecipitation}

Eight $\mu \mathrm{g}$ of anti-calpain- 2 antibody were added to $100 \mu \mathrm{L} / \mathrm{each}$ sample magnetic Dynabeads M-280 and incubated two hours on a rocking platform at room temperature. The beads were washed three times with double volume of PBS (10 mM Na-phosphate, $0.15 \mathrm{M} \mathrm{NaCl}, \mathrm{pH} 7.4$ ). The antibody was cross-linked using $20 \mathrm{mM}$ dimethyl pimelimidate dihydrochloride (DMP; Sigma-Aldrich) and $0.2 \mathrm{M}$ triethanolamine ( $\mathrm{pH}$ 8.2, Sigma-Aldrich) according to the manufacturer's product description. The cross-linked beads were washed twice in PBS and blocked with Roti-Block (Carl Roth) for one hour on a rocking platform at room temperature. Antibody-conjugated beads and Tween 20 (final concentration in the sample: $0.025 \%$ ) were added to $50 \mu \mathrm{L}$ of brain protein extract. Samples were incubated overnight on a rocking platform at $4^{\circ} \mathrm{C}$. The magnetic beads/sample solution was transferred to a magnetic particle processor (KingFisher, Thermo Fisher Scientific) (tube 1). The following three wash steps (tubes 2-4) were conducted for $10 \mathrm{~s}$ in $1 \mathrm{~mL}$ of each washing buffer: (tube 2) $0.025 \%$ Tween 20 in PBS, (tube 3) PBS and (tube 4) $50 \mathrm{mM}$ ammonium hydrogen carbonate (pH 8.0). The content of tube 4 was split in two tubes, put on a magnet and the supernatant discarded. Thirty $\mu \mathrm{L}$ of $1 \mathrm{X}$ Tris-Glycine native sample buffer (Novex) or 1x LDS-PAGE sample buffer (Novex) + DTT was added to the beads and incubated for 30 minutes at RT (for native gel) or for 10 min at $70^{\circ} \mathrm{C}$ (for western blot), shaking $(1000 \mathrm{rpm})$. The sample was put on a magnet, the supernatant saved and the beads discarded. Samples were frozen at $-80^{\circ} \mathrm{C}$ pending gel electrophores is.

\section{Gel electrophoresis and gel overlay with FRET substrate}

Fifteen $\mu \mathrm{L}$ of brain protein homogenates (regular and calpain-2 depleted) and cell lysates from the calpain- 2 culture were mixed with $15 \mu$ L Novex Trys-Glycine native gel sample buffer and loaded on a Novex 10\% Tris-Glycine gel (all from Thermo Fisher Scientific). Electrophores is was performed using Tris-Glycine native gel-running buffer (constant V, $110 \mathrm{~V}$, two hours and 25 minutes). Calpain-2-depleted brain homogenates and SHSY5Y cell lysate (10 $\mu \mathrm{g}$ of 
protein/sample) were loaded onto Novex 4-12\% Bis-Tris gels and run with NuPage MES buffer (all Thermo Fisher Scientific) at $110 \mathrm{~V}$ for one hour. Coomassie staining was performed soaking the gel for one hour in SimplyBlue SafeStain (Thermo Fisher Scientific) and washing with ultrapure water every hour until destained.

Brain protein homogenates (regular and calpain-2 depleted) and cell lysates from the calpain-2 culture were also assessed for proteolytic fluorescent activity on gel. After electrophoresis, the gel was briefly rinsed in water and incubated in $50 \mathrm{mM}$ Tris- $\mathrm{HCl} \mathrm{pH} 7.5$ for $10 \mathrm{~min}$ with light shaking. The gel was then soaked in $5 \mu \mathrm{M}$ FRET peptide in $50 \mathrm{mM}$ Tris- $\mathrm{HCl} \mathrm{pH} 7.5,300 \mu \mathrm{M}$ $\mathrm{CaCl}_{2}$ ( $2 \mathrm{mM}$ when the sample was recombinant calpain- 2 cell pellet) for $15 \mathrm{~min}$. In parallel, the gel with the brain homogenate was also soaked with $3 \mathrm{mM}$ EDTA (calcium inhibitor) or $100 \mu \mathrm{M}$ calpain inhibitor E64-c combined with the substrate. Fluorescence was observed on a UV bench using a FITC filter set from the LAS3000 imager (Fujibio). The UV bench had an additional "Superbright" filter (Vilber Lourmat) to reduce background of non-UV light. Fluorescent bands and non-fluorescent control bands were cut out from the gel and processed for mass spectrometric analysis.

\section{Western blotting}

After electrophoresis, the native gel was equilibrated for $25 \mathrm{~min}$ in NuPage Trys-Glycine transfer buffer containing $10 \% \mathrm{MeOH}$. The proteins were transferred to a nitrocellulose membrane (\#10600001, Protran $0.2 \mu \mathrm{m}$, Amersham) in a semi-dry blot apparatus $(20 \mathrm{~V}, 60$ minutes). Blocking was performed for 1 hour using 5\% milk (Blocking Grade Blocker, NonFat Dry Milk, Cat no 170-6404, Bio-Rad) in PBS-Tween (0.05\%). After blocking, the membrane was incubated with anti-calpain-2 primary antibody (E-10, sc-373966, Santa Cruz Biotechnology) in PBS-Tween (0.05\%)-0.1\% BSA overnight at $4^{\circ} \mathrm{C}$. After three washes of ten minutes each with PBS-Tween $(0.05 \%)$, the membrane was incubated with the secondary antibody IgG kappa binding protein (m-IgGK BP) conjugated to HRP (sc-516102, Santa Cruz Biotechnology) diluted 1/5000, for one hour. After another wash cycle, signals by ECL (\#RPN2235, GE Healthcare) were detected using a LAS3000 camera (Fujifilm). Western blot, antibody incubation and membrane development for other brain homogenates and SHSY5Y cell lysate were performed as above after gel equilibration in NuPage transfer buffer (Thermo Fisher Scientific) for 25 minutes. Image analysis and comparison of the optical densities (ODs) of the bands were performed with Image $J$ (Fiji). OD values for each sample were normalised against the OD of the housekeeping protein GAPDH (GAPDH Antibody-HRP (2D4A7), Novus Biologicals $\left.{ }^{\circledR}\right)$.

\section{Mass spectrometry analysis of proteins from in-gel trypsination}

NanoLC coupled to ESI high resolution hybrid quadrupole-orbitrap MS was performed with a Dionex 3000 system and a Q Exactive (both Thermo Fisher Scientific, Inc.) in a similar way as previously published [15]. Native gel cut-out bands were processed according to a standard protocol (reduction with DTT, followed by alkylation of reactive cysteines with iodoacetamide and digestion with trypsin), and samples were loaded onto an Acclaim PepMap C18 trap column (length $20 \mathrm{~mm}$, internal diameter $75 \mu \mathrm{m}$, particle size $3 \mu \mathrm{m}$, pore size $100 \AA$, Thermo Fisher Scientific, Inc.) for desalting and clean-up. Sample loading buffer was $0.05 \%$ trifluoroacetic acid in water. Separation was performed using a reversed-phase Acclaim PepMap C18 analytical column (length $150 \mathrm{~mm}$, internal diameter $75 \mu \mathrm{m}$, particle size $2 \mu \mathrm{m}$, pore size $100 \AA$, Thermo Fisher Scientific, Inc.) at a flow rate of $300 \mathrm{~nL} / \mathrm{min}$ by applying a 50 
min long linear gradient from $3 \%$ to $40 \% \mathrm{~B}$. Buffer A was $0.1 \%$ formic acid in water and buffer B was $0.1 \%$ formic acid $/ 84 \%$ acetonitrile in water. The mass spectrometer was operated in data-dependent mode, i.e., acquiring fragment mass spectra whenever the acquisition software detected peptide peaks above threshold intensity. Database searches were performed using Proteome Discoverer 2.1.1.21 (Thermo Fisher Scientific, Inc.) and Mascot search engine 2.6.1 (Matrix Science) against the databases SwissProt 2018_01 and UniProt_isoforms 20180524.

\section{Simoa assay measuring tau N-224}

The immunoassay (tau N-224) detecting fragments going from the N-terminal (antibody binding region: aa 9-18) to aa 224 was developed using Single molecule array (Simoa, Quanterix, Lexington, MA, USA) technology. Magnetic beads (Quanterix, Lexington, MA, USA) were conjugated with the capture antibody anti-Tau 224 at $0.3 \mathrm{mg} / \mathrm{mL}$ according to bead supplier's conjugation protocol. Prior to each run, Tau 224 recombinant protein calibrator was serially diluted and the biotin-labeled detection antibody Tau 12 (Nordic Biosite) was diluted to $0.5 \mu \mathrm{g} / \mathrm{mL}$ in PBS-Tween with $1 \%$ BSA. For each determination, 140000 assay beads were washed and resuspended in $100 \mu \mathrm{L}$ of CSF/brain extract, quality control sample or calibrator. After a washing step $20 \mu \mathrm{L}$ of detection antibody was added followed by 30 -minute incubation. After a final wash beads were resuspended in $100 \mu \mathrm{L}$ streptavidin-conjugated $\beta$-galactosidase (SBG, Quanterix, Lexington, MA, USA) at $150 \mathrm{pM}$ diluted in SBG Diluent (Quanterix, Lexington, MA, USA). Following five minutes of incubation, the beads were washed and transferred together with resorufin-D-galactopyranoside substrate (RGP, Quanterix, Lexington, MA, USA) to an array of wells, each well only big enough to contain one bead. The array was imaged with a charge-coupled device (CCD) camera imaging system and the images were used to differentiate between beads with and without bound analyte, giving a signal expressed as average enzyme per bead (AEB). To extract concentrations from AEBs, each sample AEB was fitted to a four-parameter logistic curve plotted from the known concentrations of the Tau 224 calibrator run in parallel with the samples. Calibrator points were run in triplicates while samples were run in duplicates.

\section{Results}

\section{Brain protein extract and cell lysate from calpain-2 cell culture (but not calpain-1) cleave the FRET substrate}

Cleavage of the substrate was observed as increased relative fluorescence over time for calpain2 but not calpain-1 (Fig. 1). Trypsin was used in this experiment as a positive control to attest the quality of the custom FRET peptide and the steep increase in RFU measured over time confirmed that the substrate could be cleaved at lysine residues (Fig. 1d). Four out of six brain protein extracts, namely CTRL 1 TBS, AD 2, CTRL 2 and CTRL 3, showed an increase in fluorescence over time, with percentages of RFU increase from baseline to the XX time point of $40.2 \%, 16.9 \%, 39.2 \%$ and $59.6 \%$, respectively (Fig. 1a,b). Cell lysates from cultures expressing both the dual vector CAPNS1/CAPN2 and CAPN2 showed fluorescent activity with a \% increase of RFU at endpoint of $59.6 \%$ (81\% for the purified form) and 39.2\%, respectively (Fig. 1c). Supernatants from the same cultures showed no activity. No activity was observed in cell culture supernatants and lysate expressing transfected with an empty vector, excluding the possibility of an artefact from bacterial proteases cleavage. Linear and non-linear models of rate of change $(\%)$ from baseline to endpoint over time show the best fit for crude and purified CAPNS1/CAPN2 (Linear: $\mathrm{R}^{2}=0.97, \mathrm{p}<0.0001$; non-linear: $\left.\mathrm{R}^{2}=0.99\right)$ and CAPN2 $\left(\mathrm{R}^{2}=0.72\right.$, $\mathrm{p}<0.0001$; non-linear: $\mathrm{R}^{2}=0.96$ ) compared to calpain-1 (Fig. 2a,b). 


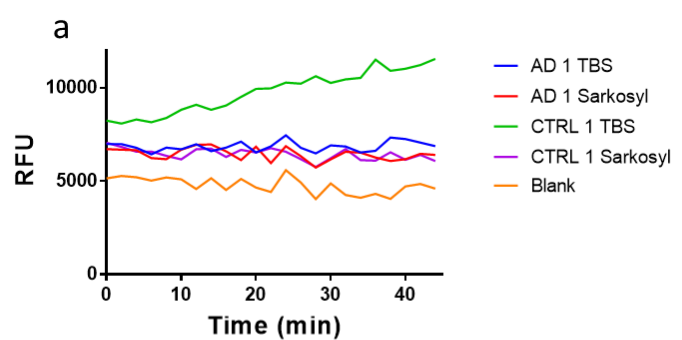

C

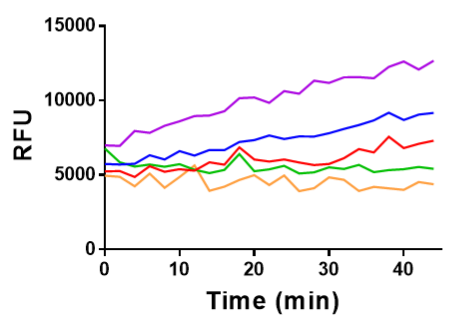

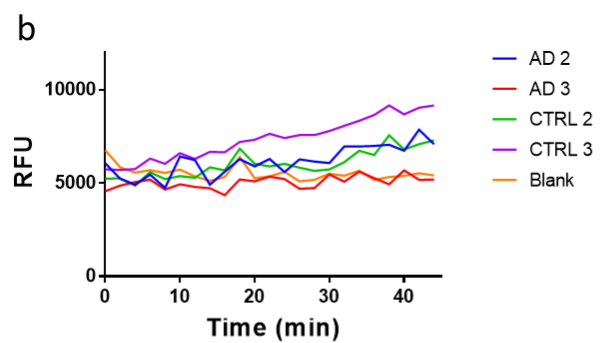

d

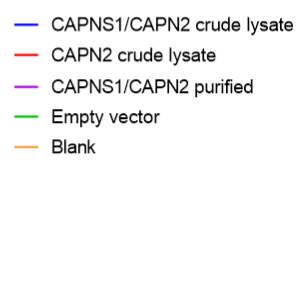

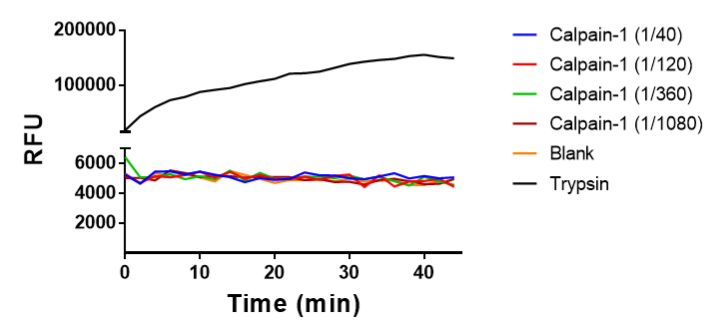

Fig. 1: Development of fluorescence from cleavage of the FRET substrate over time. a) Brain fractions extracted with protocol 1.b) Brain fractions extracted with protocol 2.c) Recombinant calpain- 2 crude and purified lysates. d) Recombinant calpain-1 dilutions. Y-axes show relative fluorescence units. $\mathrm{X}$-axes show time in minutes.
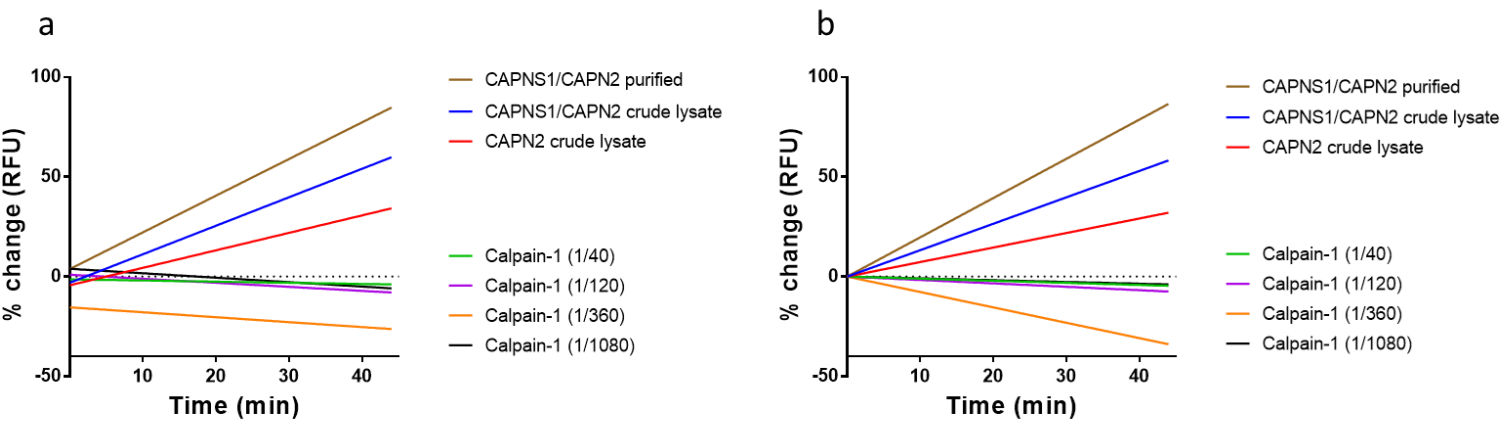

Fig. 2: linear (a) and non-linear (b) model of rate of change (\%) in RFU from baseline to endpoint of the measurement. Explain axes.

\section{Brain extract and cell lysate from calpain-2 culture show proteolytic activity on native gel overlaid with FRET substrate}

Brain extracts showing clevage of the quenched FRET peptide (CTRL 1 TBS, CTRL 3) and AD 1 TBS were run on a native gel and soaked with a solution containing the FRET peptide. The same approach was used for cell lysates from calpain-2 culture (both CAPNS1/CAPN2 and CAPN2) and empty vector culture. A band of fluorescent activity was observed at a molecular weight (MW) of $\sim 80 \mathrm{kDa}$ in CTRL brains and calpain-2 lysates, but not in the empty vector lysate (Fig. 3). A band at the same MW was also observed at Coomassie stain of the brain lysate (Fig. 3). 


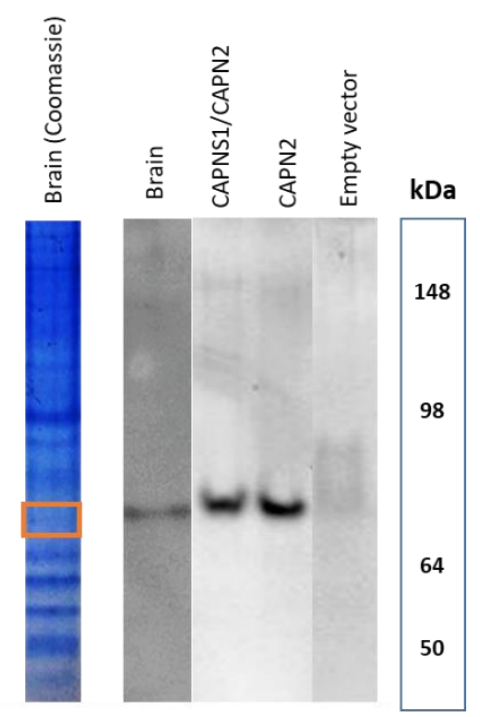

Fig. 3: Coomassie stain and native gel of brain extract (lanes 1-2). Western blot of calpain-2 and empty vector cell lysate (lanes 3-5). The molecular weight marker is indicated to the right of the panel.

In-gel trypsination of brain-derived band with proteolytic activity reveals presence of calpain-2 catalytic subunit, but not calpain-1

The active band from CTRL 1 TBS and the correspondent gel region of the AD brain were trypsinated in-gel and analysed with MS, showing a total of 603 and 397 protein hits, respectively. The catalytic and regulatory subunit of calpain-2 (CAPN2, CAPNS1) were present in both bands. In the active band from CTRL 1 TBS, 22 CAPN2 peptides were detected spanning almost the full protein sequence with a coverage of $37 \%$ (Fig. 4). In the same sample, 8 peptides covering $51 \%$ of the calpain- 1 were detected (Fig. 4). No calpain-1 peptides were detected in any of the catalytically active sample bands.

Calpain-2 catalytic subunit OS=Homo sapiens $\mathrm{GN}=\mathrm{CAPN} 2 \mathrm{PE}=1 \mathrm{SV}=6$

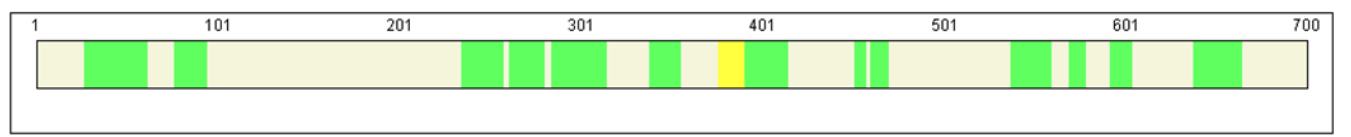

Coverage: $37 \%$

Calpain small subunit 1 OS=Homo sapiens GN=CAPNS1 PE=1 SV=1

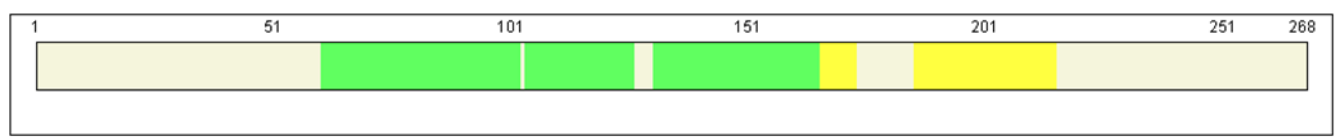

Coverage: $51 \%$

Fig. 4: MS sequence coverage of calpain-2 subunits from trypsin-digested gel bands.

\section{Calpain-2 depletion from brain and chemical inhibition suppress proteolysis of the FRET substrate}

Brain homogenates immunodepleted of calpain-2 (CTRL 1 TBS, CTRL 3) showed no proteolytic activity on the FRET substrate in the native gel (Fig. 5). The calcium inhibitor 
EDTA could not suppress calpain activity in the brain homogenate, while a calpain inhibitor (E64c) inhibited the proteolytic activity of the brain on the FRET substrate (Fig. 5).

Western blot showed bands positive for calpain-2 at $80 \mathrm{kDa}$ (corresponding to the MW of the fluorescent band on the gel) and smaller MW ( 55 kDa, $\sim 45 \mathrm{kDa})$ in CTRL 1 TBS and CTRL 3 homogenates (Fig. 6). The smaller bands disappeared in the calpain-2-depleted samples and the intensity of the $80 \mathrm{kDa}$ band was reduced (-16.5\% in CTRL 1, $-15.1 \%$ in CTRL 3) (Fig. 6). The smaller bands were present in the eluate from the beads cross-linked to anti-calpain-2 antibody, while there was no unspecific binding of the protein to naked beads (Fig. 6).

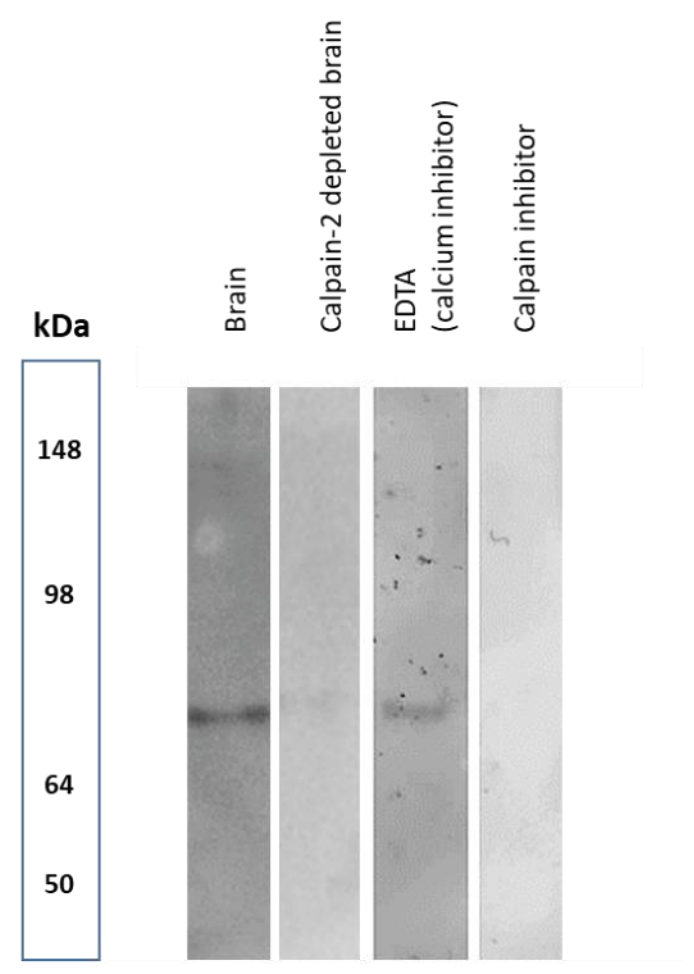

Fig. 5: Brain extract on native gel overlaid with FRET substrate. Calpain-2-depleted brain extract and brain extract combined with calpain inhibitor show no cleavage of the FRET substrate.
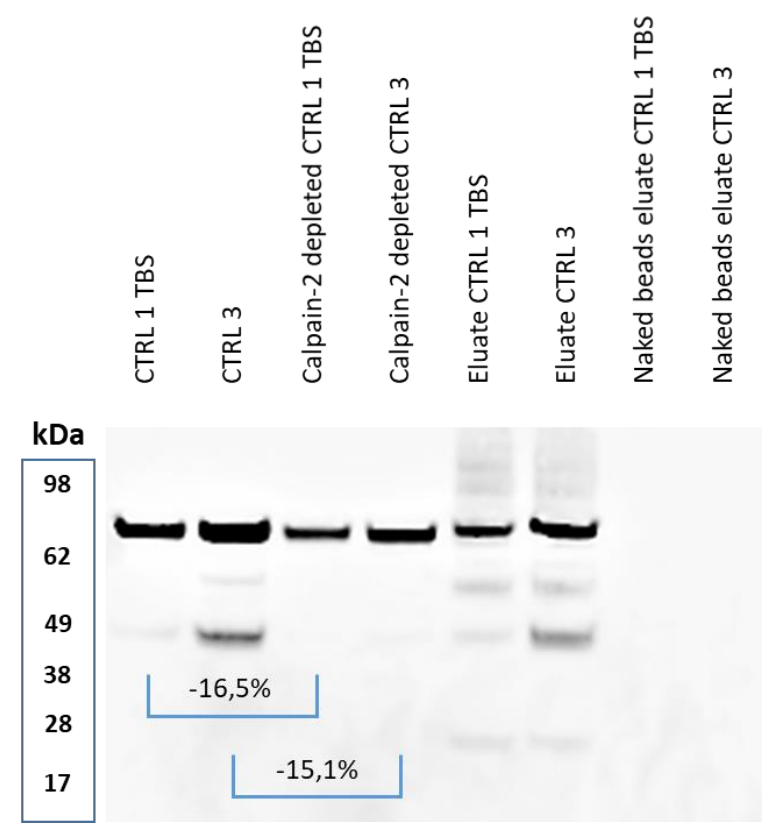
Fig. 6: IP on brain samples with anti-calpain-2 antibody. The calpain-2-depleted samples show a reduction in OD of $16.5 \%$ and $15.1 \%$.

\section{Gene knock-down of calpain-2 in SHSY5Y cells results in lower levels of N-224 tau in cell media}

Using, siRNA, calpain-2 was successfully knocked-down in SHSY5Y cells, as shown at western blot with anti-calpain-2 antibody by a reduction in OD (normalised against GAPDH) of $93 \%$ compared to the scrambled sample (Fig. 7a). Cell media from the same samples showed a reduction in the concentration of N-224 tau of $75 \%$ compared to the cell media from the scrambled sample (Fig. 7b). In order to examine the gene specificity of the siRNA and investigate a possible compensation by calpain-1, we measured the gene expression of CAPN1, the gene codes for calpain-1, and CAPN2, the gene codes for calpain-2, in CAPN2-siRNA treated cells (Fig. 8). After the siRNA treatment, mRNA level of CAPN2 significantly decreased as compared to the scrambled control (Fig. 8a); CAPN1, yet, remained stable (Fig. $8 \mathrm{~b})$. The gene expression data suggests that the decreased tau 224 in the conditioned cell media was indeed due to decreased level of CAPN2 by the siRNA. Moreover, decreased level of CAPN2 mRNA and tau 224 did not cause any compensatory reaction on CAPN1.

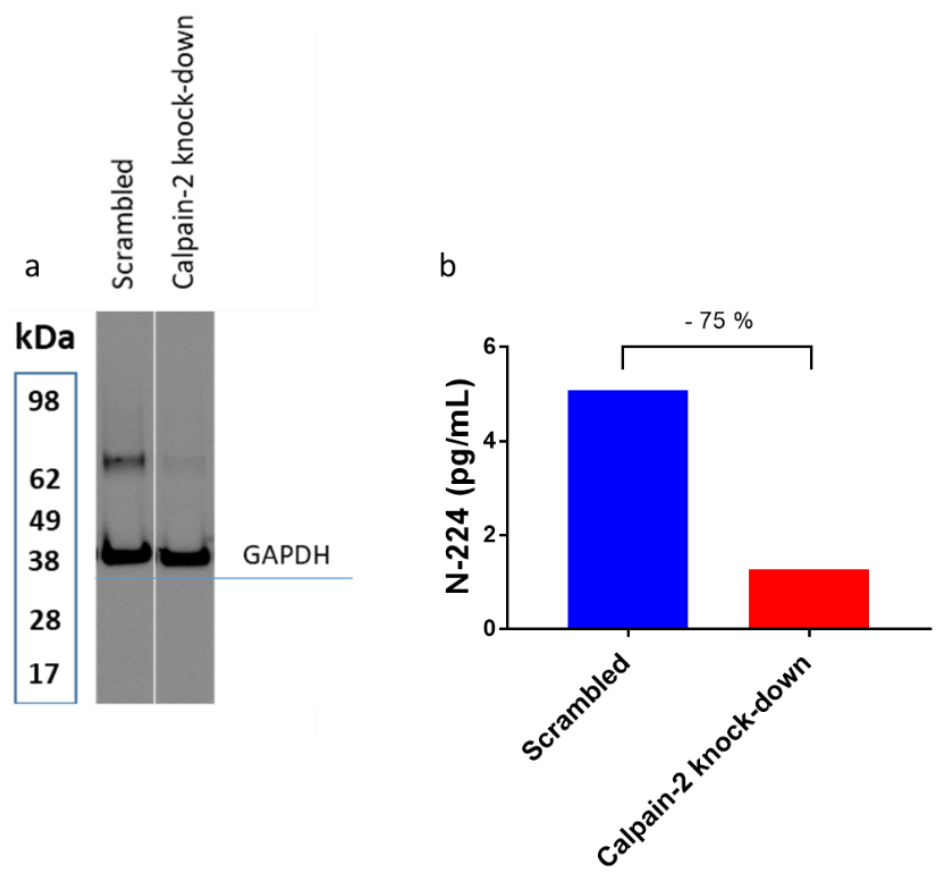

Fig. 7: a) Western blot on lysate from SHSY5Y cells with anti-calpain-2 antibody; b) N-224 tau concentrations in cell media from scrambled and calpain-2 knock-down cells.

a

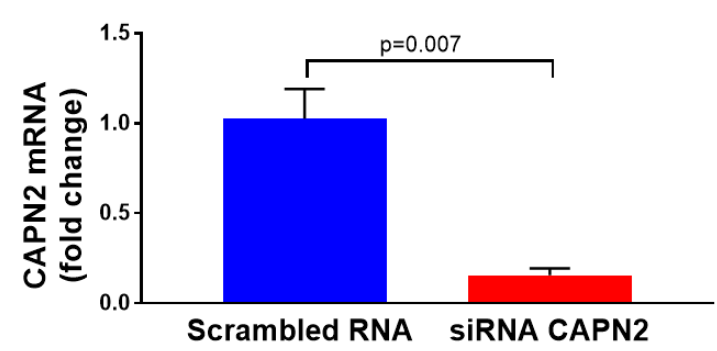

b

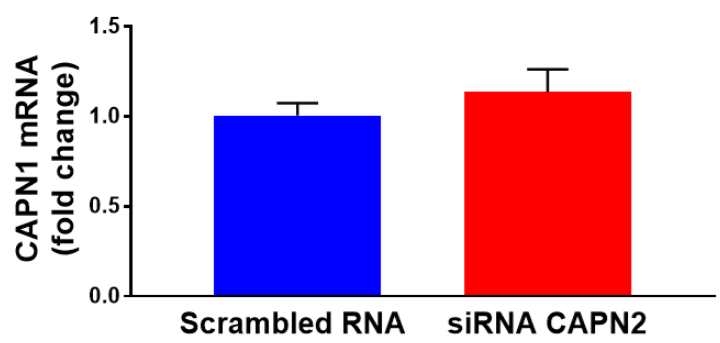


Fig. 8: change in CAPN2 (a) and CAPN1 (b) mRNA expression in scrambled and after calpain2 knock-down cells.

\section{Discussion}

A large body of literature has shown that tau protein undergoes extensive proteolysis and is present in CSF as a series of fragments [5-16]. Our group has shown that a major CSF pool of tau ends at aa 224, and the measurement of fragments spanning N-224 might have a diagnostic and prognostic role in tauopathies [16]. Since the enzyme responsible for the cleavage at aa 224 is unknown, but calpain cleavage has been previously observed in regions surrounding our site of interest, the aim of the present study was to evaluate if calpains could be responsible for the production of the N-224 fragment [18-20].

We first tested a commercially available recombinant form of calpain-1, combined with a FRET substrate containing a tau sequence of 10 aa, including aa 224, following a procedure previously tested by our group [37]. If cleavage had occurred, the fluorophore at the $\mathrm{N}$-terminus of the substrate would have been freed by the action of quencher at the $\mathrm{C}$-terminal, and have shown an increase in fluorescent activity when measured on a spectrophotometer. Although we tested different concentrations of the enzyme and added $\mathrm{CaCl}_{2}$ to the substrate to fulfil the calcium requirements for activation of calpain-1 ( $\mu$-calpain), none of the combination showed cleavage of the FRET substrate.

We then hypothesize that calpain-2 could be a candidate for cleavage, as the literature had previously suggested calpain- 2 as the proteases responsible for cleavage at aa 230 [19]. As recombinant calpain-2 was not commercially available at the time of our study, we produced our own recombinant form using a dual vector expressing either the regulatory and catalytic subunit of the enzyme combined (CAPNS1/CAPN2) or only the catalytic subunit (CAPN2). Cell lysates (crude and purified) from both cultures showed proteolytic activity on the FRET substrate, while the lysate from an empty vector-transfected cell culture did not, excluding any false positives coming from bacterial proteases. Also, the fluorescent activity could not be caused by calpain- 2 cleavage at the previously described site aa 230 , since this aa was not included in the sequence of the custom made FRET peptide (aa 220-228) [18, 19].

In parallel, we also tested brain protein extracts in combination with the FRET substrate, following the same procedure, and selected the ones that showed the highest rate of proteolytic activity for further analysis. In this experiment, the proteolytic activity appeared somewhat higher in control brains rather than $\mathrm{AD}$, although one $\mathrm{AD}$ brain showed an increase in fluorescence of $16.9 \%(\mathrm{AD} 2)$. The enzyme also seem to be contained in the soluble fraction of brain proteins (TBS), as no activity was observed in the sarkosyl-soluble fractions. However, we cannot draw conclusions in term of disease-specificity of the cleavage due to several limitations, e.g. the small sample size of the brains in exam, the different brain regions to which the samples belonged and the different extraction techniques. The brains that showed the most increase in fluorescence from baseline to endpoint were run on a native gel and overlaid with a solution containing the FRET substrate; the aim was to observe and isolate the active band where the enzyme responsible for the cleavage would run. The band that showed fluoresce nce on the gel was cut out and digested with trypsin prior to MS analysis. As expected, the band contained a large number of brain proteins, but we confirmed the presence of both the regulatory and catalytic subunit of calpain-2 at this site. Although the AD brain showed no activity on the gel, calpain-2 was present at the corresponding gel band when analysed with MS, with fewer peptide hits. It is possible that the lower calpain-2 concentration in this specific sample caused the absence of observable proteolytic activity. Cell lysates from calpain-2 culture also confirmed their activity on the native gel, producing a band at the same MW as the brains. 
When depleting calpain-2 from the brains that showed proteolytic activity, the cleavage of the FRET substrate was suppressed. We then performed western blot on the same samples using the same antibody that was crosslinked to the beads used for IP. The image analysis showed that the IP was efficient, lowering the content of calpain-2 in the sample up to $\sim 16 \%$. Smaller fragments of calpain- 2 were also observed in the samples, belonging to the $\mathrm{N}$-terminal region of the protein (antibody epitope: aa 2-27). These fragments are likely to be the result of the activation of calpain- 2 caused by $\mathrm{CaCl}_{2}$; it has been shown that once calpain- 2 is activated, it undergoes autoproteolysis starting from the $\mathrm{N}$-terminus of the protein (N-end rule), which also contributes to lower the levels of calcium required for maximal activation [38-42]. However, adding a calcium inhibitor (EDTA) to the FRET substrate did not suppress its proteolytic activity, possibly because quenching calcium cannot revert the activation of calpain- 2 once it has occurred. A calpain inhibitor, however, managed to suppress the activity of the enzyme.

Calpain-2 gene knock-down in SHSY5Y cells was successful, showing a significant reduction in the expression of CAPN2 ( $\mathrm{p}=0.007)$. CAPN2 knock-down did not interfere with the expression of CAPN1, excluding calpain-1 as responsible for the cleavage at aa 224. In cell media from calpain-2 knock-down SHSY5Y cells, N-224 tau showed a 75\% reduction compared to the scrambled control media. Since the N-224 tau assay binds exclusively tau fragments ending at aa 224, these results suggest that knock-down of calpain-2 is directly related to the decrease in N-224 tau, and possibly responsible for cleavage of the protein at this site. Although we cannot exclude, at this stage, that other factors act downstream of the calpain2 knock-down, the drastic reduction of N-224 in the media and the specificity of the N-224 assay strongly link the enzyme activity to the production of this specific fragment.

In conclusion, we have shown that calpain- 2 cleaves tau between aa 220 and aa 228, and calpain-2 knockdown reduces significantly the amount of tau ending at aa 224 in cell media. Calpain upregulation has been linked to neurodegenerative diseases, and N-224 tau has been shown as a good biomarker for tauopathies. Taken together, these findings encourage further investigation of the calpain-2 pathway, in order to elucidate the role of tau fragmentation in neurodegeneration and for discovery of new targets in tauopathy treatment.

\section{Acknowledgements}

The work was supported by grants from the Swedish Research Council (\#2018-02532), the European Research Council (\#681712), Swedish State Support for Clinical Research (\#ALFGBG-720931), the Knut and Alice Wallenberg Foundation, Frimurarestiftelsen, Stiftelsen för Gamla Tjänarinnor, Alzheimerfonden, Hjärnfonden, xxx...

\section{Conflicts of interest}

HZ has served at scientific advisory boards for Roche Diagnostics, Wave, Samumed and CogRx, has given lectures in symposia sponsored by Biogen and Alzecure, and is a co-founder of Brain Biomarker Solutions in Gothenburg AB, a GU Ventures-based platform company at the University of Gothenburg (outside submitted work).

\section{References}


1. Biernat, J., et al., Phosphorylation of Ser262 strongly reduces binding of tau to microtubules: distinction between PHF-like immunoreactivity and microtubule binding. Neuron, 1993. 11(1): p. 153-63.

2. Simic, G., et al., Tau Protein Hyperphosphorylation and Aggregation in Alzheimer's Disease and Other Tauopathies, and Possible Neuroprotective Strategies. Biomolecules, 2016. 6(1): p. 6.

3. Wang, Y.P., et al., Stepwise proteolysis liberates tau fragments that nucleate the Alzheimerlike aggregation of full-length tau in a neuronalcell model. Proc Natl Acad Sci U S A, 2007. 104(24): p. 10252-7.

4. Quinn, J.P., et al., Tau Proteolysis in the Pathogenesis of Tauopathies: Neurotoxic Fragments and Novel Biomarkers. J Alzheimers Dis, 2018. 63(1): p. 13-33.

5. Johnson, G.V., et al., The tau protein in human cerebrospinal fluid in Alzheimer's disease consists of proteolytically derived fragments. J Neurochem, 1997. 68(1): p. 430-3.

6. Meredith, J.E., Jr., et al., Characterization of novel CSF Tau and ptau biomarkers for Alzheimer's disease. PLoS One, 2013. 8(10): p. e76523.

7. Amadoro, G., et al., Cerebrospinalfluid levels of a 20-22 kDa NH2 fragment of human tau provide a novel neuronal injury biomarker in Alzheimer's disease and otherdementias. J Alzheimers Dis, 2014. 42(1): p. 211-26.

8. Barthelemy, N.R., et al., Tau Protein Quantification in Human Cerebrospinal Fluid by Targeted Mass Spectrometry at High Sequence Coverage Provides Insights into Its Primary Structure Heterogeneity. J Proteome Res, 2016. 15(2): p. 667-76.

9. Russell, C.L., et al., Comprehensive Quantitative Profiling of Tau and Phosphorylated Tau Peptides in Cerebrospinal Fluid by Mass Spectrometry Provides New Biomarker Candidates. J Alzheimers Dis, 2017. 55(1): p. 303-313.

10. Hansson, K.T., et al., Expanding the cerebrospinalfluid endopeptidome. Proteomics, 2017. 17(5).

11. Barthelemy, N.R., et al., Differential Mass Spectrometry Profiles of Tau Protein in the Cerebrospinal Fluid of Patients with Alzheimer's Disease, Progressive Supranuclear Palsy, and Dementia with Lewy Bodies. J Alzheimers Dis, 2016. 51(4): p. 1033-43.

12. Sato, C., et al., Tau Kinetics in Neurons and the Human Central Nervous System. Neuron, 2018. 97(6): p. 1284-1298 e7.

13. Borroni, B., et al., Pattern of Tau forms in CSF is altered in progressive supranuclear palsy. Neurobiol Aging, 2009. 30(1): p. 34-40.

14. Borroni, B., et al., Tau forms in CSF as a reliable biomarkerfor progressive supranuclearpalsy. Neurology, 2008. 71(22): p. 1796-803.

15. Chen, Z., et al., Learnings about the complexity of extracellular tau aid development of a blood-based screen for Alzheimer's disease. Alzheimers Dement, 2018.

16. Cicognola, C., et al., Novel tau fragments in cerebrospinal fluid: relation to tangle pathology and cognitive decline in Alzheimer's disease. Acta Neuropathologica, 2018.

17. Ferreira, A. and E.H. Bigio, Calpain-mediated tau cleavage: a mechanism leading to neurodegeneration shared by multiple tauopathies. Mol Med, 2011. 17(7-8): p. 676-85.

18. Garg, S., et al., Cleavage of Tau by calpain in Alzheimer's disease: the quest for the toxic $17 \mathrm{kD}$ fragment. Neurobiol Aging, 2011. 32(1): p. 1-14.

19. Park, S.Y. and A. Ferreira, The generation of a $17 \mathrm{kDa}$ neurotoxic fragment: an alternative mechanism by which tau mediates beta-amyloid-induced neurodegeneration. J Neurosci, 2005. 25(22): p. 5365-75.

20. Matsumoto, S.E., et al., The twenty-four KDa C-terminal tau fragment increases with aging in tauopathy mice: implications of prion-like properties. Hum Mol Genet, 2015. 24(22): p. 640316.

21. Dear, T.N. and T. Boehm, Identification and characterization of two novel calpain large subunit genes. Gene, 2001. 274(1-2): p. 245-52. 
22. Moldoveanu, T., et al., $A \mathrm{Ca}(2+)$ switch aligns the active site of calpain. Cell, 2002. 108(5): $\mathrm{p}$. 649-60.

23. Hanna, R.A., R.L. Campbell, and P.L. Davies, Calcium-bound structure of calpain and its mechanism of inhibition by calpastatin. Nature, 2008. 456(7220): p. 409-12.

24. Goll, D.E., et al., The calpain system. Physiol Rev, 2003. 83(3): p. 731-801.

25. Baudry, M. and X. Bi, Calpain-1 and Calpain-2: The Yin and Yang of Synaptic Plasticity and Neurodegeneration. Trends Neurosci, 2016. 39(4): p. 235-245.

26. Chesser, A.S., S.M. Pritchard, and G.V. Johnson, Tau clearance mechanisms and their possible role in the pathogenesis of Alzheimerdisease. Front Neurol, 2013. 4: p. 122.

27. Vosler, P.S., C.S. Brennan, and J. Chen, Calpain-mediated signaling mechanisms in neuronal injury and neurodegeneration. Mol Neurobiol, 2008. 38(1): p. 78-100.

28. Kurbatskaya, K., et al., Upregulation of calpain activity precedes tau phosphorylation and loss of synaptic proteins in Alzheimer's disease brain. Acta Neuropathol Commun, 2016. 4: p. 34.

29. Rao, M.V., et al., Specific calpain inhibition by calpastatin prevents tauopathy and neurodegeneration and restores normal lifespan in tau P301L mice. J Neurosci, 2014. 34(28): p. 9222-34.

30. Lewis, J., et al., Neurofibrillary tangles, amyotrophy and progressive motor disturbance in mice expressing mutant (P301L) tau protein. Nat Genet, 2000. 25(4): p. 402-5.

31. Yang, L.S. and H. Ksiezak-Reding, Calpain-induced proteolysis of normal human tau and tau associated with paired helical filaments. Eur J Biochem, 1995. 233(1): p. 9-17.

32. Siklos, M., M. BenAissa, and G.R. Thatcher, Cysteine proteases as therapeutic targets: does selectivity matter? A systematic review of calpain and cathepsin inhibitors. Acta Pharm Sin B, 2015. 5(6): p. 506-19.

33. Braak, H. and E. Braak, Neuropathologicalstageing of Alzheimer-related changes. Acta Neuropathol, 1991. 82(4): p. 239-59.

34. Hata, S., et al., Efficient expression and purification of recombinant human $m$-calpain using an Escherichia coli expression system at low temperature. J Biochem, 2012. 151(4): p. 417-22.

35. McCartney, C.E. and P.L. Davies, Bacterial Expression and Purification of Calpains. Methods Mol Biol, 2019. 1915: p. 13-27.

36. Petra Bergström, L.A., Faisal Hayat Nazir, Tugce Munise Satir, Jamie Toombs, Henrietta Wellington, Joakim Strandberg, Thomas Olsson Bontell, Hlin Kvartsberg, Maria Holmström, Cecilia Boreström, Stina Simonsson, Tilo Kunath, Anders Lindahl, Kaj Bl, Amyloid precursor protein expression and processing are differentially regulated during cortical neuron differentiation. Scientific Reports 2016.

37. Becker, B., et al., Alzheimer-associated cerebrospinal fluid fragments of neurogranin are generated by Calpain-1 and prolylendopeptidase. 2018. 13(1): p. 47.

38. Baki, A., et al., Autolysis parallels activation of mu-calpain. Biochem J, 1996. 318 ( Pt 3): p. 897-901.

39. Elce, J.S., C. Hegadorn, and J.S. Arthur, Autolysis, Ca2+ requirement, and heterodimer stability in m-calpain. J Biol Chem, 1997. 272(17): p. 11268-75.

40. Melloni, E., et al., Modulation of the calpain autoproteolysis by calpastatin and phospholipids. Biochem Biophys Res Commun, 1996. 229(1): p. 193-7.

41. Murphy, R.M., E. Verburg, and G.D. Lamb, Ca2+activation of diffusible and bound pools of mu-calpain in rat skeletal muscle. J Physiol, 2006. 576(Pt 2): p. 595-612.

42. Piatkov, K.I., et al., Calpain-generated natural protein fragments as short-lived substrates of the N-end rule pathway. Proc Natl Acad Sci U S A, 2014. 111(9): p. E817-26. 